Patient $\times$ is a 3 year old male who had neonatal hypoglycaemia and confirmed homozygosity for the GSD Type IIIa gene. Patient $\times$ was commenced on the KD at 8 months of age when progression of his GSD resulted in severe cardiomyopathy. The following outlines the transition onto the $\mathrm{KD}$ and clinical findings between 8-22 months

Method Standard dietetic treatment was provided from birth to maintain euglycaemia along with placement of a percutaneous endoscopic gastrostomy. Continuous 24 hour feeding was required due to unsuccessful bolus feeding. A modified KD incorporating MCT fat -which was has been noted to further aid ketosis was commenced at 8 months with a ketogenic ratio of $0.5: 1$ building to $1: 1$ within 2 weeks. Ketones and blood sugar levels were closely monitored with a threshold of $2.6 \mathrm{mmol} / \mathrm{L}$ of glucose and ketones $>1 \mathrm{mmol} / \mathrm{L}$ before hypoglycaemia intervention was required.

Results Prior to the KD, there was a high glucose infusion rate (GIR) of $9.75 \mathrm{mg}$ glucose $/ \mathrm{kg} /$ minute. After initiation of the $\mathrm{KD}$, the GIR reduced initially to $5.8 \mathrm{mg}$ glucose $/ \mathrm{kg} /$ minute, with a gradual increase of MCT fat from $6 \%$ to $28 \%$ and the GIR further reduced to $2.73 \mathrm{mg}$ glucose $/ \mathrm{kg} /$ minute. There were no episodes of hypoglycaemia and ketones ranged from 1- $2.9 \mathrm{mmol} / \mathrm{L}$. Echocardiographs showed a significant improvement in cardiac function with a cardiac output reduction of 137 to $39 \mathrm{mmHg}$.

Discussion The KD was trialled as an alternative treatment. It resulted in the reduced intake of carbohydrate and the subsequent reduction of glycogen build-up within cardiac muscle. Ketones were used as an alternative fuel source and euglycaemia was maintained

Conclusion The KD should be considered as an alternative treatment for GSD Type IIIa where standard intervention is not effective.

\section{GP230 FETAL ACETYLCHOLINE RECEPTOR INACTIVATION DUE TO MATERNAL MYASTHENIA GRAVIS: AN UNDERRECOGNISED, DEVASTATING BUT POTENTIALLY PREVENTABLE AND TREATABLE DISORDER}

\begin{abstract}
${ }^{1}$ Mark O'Rahelly*, ${ }^{2}$ Andreas Hahn, ${ }^{3}$ Cam-Tu Nguyen, ${ }^{4}$ Dae-Seong Kim, ${ }^{5}$ Shin Y Byun, ${ }^{6}$ Ulrike Schara, ${ }^{6}$ Maria Henrich, ${ }^{7,8,9}$ Jacob Leslie, ${ }^{7,8,9}$ Angela Vincent, ${ }^{1}$ Nicholas M Allen*, 10,11,12 Heinz Jungbluth*. 'Department of Paediatrics, Galway University Hospital/National University of Ireland, Galway, Ireland; ' ${ }^{2}$ Department of Child Neurology, Feulgenstr, Giessen, Germany; ${ }^{3}$ Clinical Neurological Sciences, Children's Hospital, London Health Sciences Centre, London, Ontario, Canada; ${ }^{4}$ Department of Neurology, Pusan National University School of Medicine, Pusan, Korea, Republic of; ${ }^{5}$ Department of Paediatrics, Pusan National University School of Medicine., Pusan, Korea, Republic of; ${ }^{6}$ Department of Paediatric Neurology, Developmental Neurology and Social Paediatrics, University of Essen, Essen, Germany; ${ }^{7}$ Department of Clinical Neurology, Oxford University, Oxford, UK; ${ }^{8}$ Department of Clinical Neurology, John Radcliffe Hospital, Oxford, UK; ${ }^{9}$ Department of Clinical Neurosciences, Weatherall, Institute of Molecular Medicine, University of Oxford, Oxford, UK; ${ }^{10}$ Department of Paediatric Neurology, Neuromuscular Service, Evelina's Children Hospital, Guy's and St. Thomas' Hospital NHS Foundation Trust, London, UK; ${ }^{11}$ Randall Division for Cell and Molecular Biophysics, Muscle Signalling Section., London, UK; ${ }^{12}$ Department of Basic and Clinical Neuroscience, IOPPN, King's College, London, UK
\end{abstract}

\subsection{6/archdischild-2019-epa.289}

Aim Fetal acetylcholine receptor inactivation syndrome (FARIS) occurs in offspring of mothers affected by myasthenia gravis (MG), from in-utero exposure to acetylcholine receptor (AChR)-antibodies targeting the fetal AChR $\gamma$-subunit. FARIS causes damage to the fetal neuromuscular junction which is crucial in muscle development, causing a persistent myopathy. FARIS may initially be mistaken for Transient
Neonatal Myasthenia Gravis (TNMG), congenital neuromuscular disorders and one of the many causes of neonatal hypotonia. This study aimed to determine the clinical spectrum of FARIS and assess oral salbutamol as a novel pharmacological therapy.

Methods Detailed review of antenatal and postnatal clinical features in novel FARIS cases seen in international neuromuscular centres. Antibody data analysis was performed at the Oxford neuroimmunology research laboratory. Oral salbutamol was trialled in five cases based on previously reported benefit in one of our patients.

Results We identified 12 novel FARIS cases. At delivery resuscitation was required in all and intubation in nine, all had severe generalised hypotonia. Two infants with arthrogryphosis-multiplex-congenita phenotype died in the neonatal period. Among survivors, there was requirement for mechanical ventilation $(n=9)$, NIPPV $(n=2)$, oxygen $(n=1)$, and supplemental NG/PEG feeding $(n=12)$. The presence of severe generalised hypotonia with dysmorphic features prompted investigations for other neuromuscular, genetic and metabolic disorders which were negative. Common features included facial weakness $(n=12)$ and limb contractures $(n=9)$. Newly described disease features: diaphragmatic paresis $(n=5)$, hearing impairment $(n=3)$, CNS involvement $(n=3)$, pyloric stenosis $(n=2)$, extra-ocular eye restriction $(n=2)$, non-progressive scoliosis $(n=2)$, and jaw opening contracture $(n=1)$. Motor development of patients improved with time. Respiratory complications (tracheostomy;n=2), feeding difficulties (PEG;n=2), facial weakness and speech impairment (from velopharyngeal incompetence) persisted in most. TNMG treatments(immunotherapy/ pyridostigmine) were little or no benefit. Novel use of oral salbutamol improved fatiguability, ptosis, oromotor dysfunction, muscle tone, articulation and voice volume in all patients. In $8 / 12$ pregnancies maternal myasthenia gravis hadn't been established antenatally, and many mothers were pauci/asymptomatic. All had AChR-antibodies targeting the fetal $\gamma$-subunit confirming diagnosis. Where subsequent pregnancies were treated aggressively(immunotherapy), infants had improved outcomes.

Conclusions This report demonstrates and expands the phenotypic spectrum of FARIS, and emphasises oral salbutamol therapy as a potentially beneficial treatment. FARIS should be considered (mothers or infants tested for fetal specific AChR-Abs) in infants presenting with neonatal hypotonia, myopathic features and/or a suggestive antenatal history, even in the absence of a maternal MG diagnosis. Aggressive treatment with immunotherapy in pregnancy may improve outcomes.

\section{GP231 REVIEW OF INVESTIGATIONS CARRIED OUT DURING THE FIRST PRESENTATION OF ACQUIRED DEMYELINATING SYDNROMES OVER A TEN YEAR PERIOD}

Susan Harvey*, Niamh McSweeney. Cork University Hospital, Cork, Ireland

\subsection{6/archdischild-2019-epa.290}

Aims A first episode of suspected demyelination presents a diagnostic challenge often having non-specific signs which overlap with other inflammatory white matter, neurometabolic and genetic disorders. The first episode may be a presentation of acute disseminated encephalomyelitis, multiple 
sclerosis, optic neuritis, transverse myelitis or NMO spectrum disorders ${ }^{1,2}$. Associated morbidity and mortality of each is vastly different. Gaining accurate information through appropriate investigation is vital for appropriate treatment and counselling. There is currently no national or international guideline for investigation of an acute demyelinating episode.

Methods Retrospective review of all cases of a first demyelinating event over a 10 year period $(2008$ - 2018) at Cork University Hospital. Laboratory investigations, imaging and clinic letters were reviewed.

Results In total eighteen cases were reviewed. Eventual diagnoses were 7 ADEM, 4 ADEM with transverse myelitis, 4 Multiple Sclerosis and 3 Optic Neuritis. Median presentation age was 6 years (1 year 4 months - 15 years 10 months). WCC and CSF microscopy was done in 100\%. CRP and ESR done in $89 \%$ and $28 \%$ respectively. Investigations for bacterial and viral causes either on serum, CSF or swabs was inconsistent varying between $11-83 \%$. CSF antibodies, including anti MOG, anti-NMDA, Aquaporin 4 and anti voltage gated potassium channel antibodies were sent in $6-39 \%$ of cases dependant on test. Oligoclonal bands were sent in $83 \%$. Imaging was undertaken in all cases with seventeen having an MRI Brain. Median time to MR brain was 1 day (0 days -6 days). Fourteen cases had a MR spine with median time to spinal imaging of 2 days (1 day - 11 days).

Conclusion This review highlights the variable approach to investigation of suspected demyelination. The wide differential and need for prompt treatment to prevent long term neurological disability means there are multiple complex investigations required within a short time period. The laboratory investigations and neuroimaging required are labour intensive and incur significant financial cost. This is of particular importance in children, many of whom will require sedation and at times general anaesthetic to ensure successful obtaining of samples. The availability of a local protocol would guide clinicians investigation when faced with an unfamiliar presentation under significant time pressure. It would ensure appropriate and timely investigation enabling appropriate treatment and counselling.

\section{GP232 MANAGEMENT OF SEIZURES IN CHILDREN WITH THERAPY-RESISTANT EPILEPSY}

\footnotetext{
1,2Globa Oksana Valer'yevna* ${ }^{*},{ }^{1,2}$ Liudmila Kuzenkova, ${ }^{1}$ Natalia Zhourkova, ${ }^{1}$ Elena Sorokina, ${ }^{1}$ Tatiana Podkletnova, ${ }^{1}$ Natalia Andreenko, ${ }^{1}$ Kiril Savost'yanov, ${ }^{1}$ Vsevolod Pinelis. 'National Medical Research Center for Children's Health, Moscow, Russian Federation; ${ }^{2}$ Sechenov university, Moscow, Russian Federation
}

\subsection{6/archdischild-2019-epa.291}

Epilepsies in children represent a heterogeneous group of disorders and syndromes with different etiology, severity, prognosis and treatment. Early diagnosis, accurate recognition of underlying aetiologies leads to more effective management and treatment and improve overall health and quality of life. Genetic testing is very important in the cases of therapy-resistant seizures. The purpose of study was to recognise the possible reason of failed AED treatment and to find the ways to owercome it.

Methods 45 patients with different forms of epilepsy aged from 3 months to 16 years not the candidate for surgical treatment have been studied. The long duration EEG, high resolution MRI, blood biochemical tests, blood level lactate and ammonia, amino acid, organic acid and disturbance of fatty oxidation by TMS, genetic investigations (mtDNA and exome sequence), measurement of autoantibodies to NR2 and GluR1 in blood serum by ELISA were performed to these children.

Results The respiratory chain disorders confirmed by mtDNA sequence were found in 11 children. Metabolic epilepsies discovered in patients have the following origins: two with glutaric aciduria type1, one - glutaric aciduria type2, one with propionic aciduria, one with methylmalonic aciduria, one with Gaucher's disease type3, two patient with glycogenosis type 9, two patients with ceroid lipofuscinosis type 2 and 6,lysosomal storage disorders in 3 cases.

Genetic epilepsies with mutation in genes SCN8A (two patients), GRIN2A, KCNMA1, SRPX2, SCN9A, ACO2, ARHGEF9, 15q11.2q13.3,TSC- 4 patients were revealed. In other cases with normal MRI the reason of pharmacoresistant seizures was not discover yet. The elevated level of autoantibodies to glutamate NR2 and GluR1 receptors were found in children of these groups. But in patients with metabolic epilepsies the elevation level of autoantibodies to NR2 was in 4 to 7 times higher in comparing with children with genetic epilepsies. In children with metabolic disorders and energy metabolism disorders we use the specific therapy (special diet, L-carnitine, vitamins, enzyme replacement therapy etc) in cases which it possible, avoid valproic acid in treatment of children with mitochondrial disorders and glutaric acidurias, as well we use the phenytoin in patient with potassium channel mutation SCN8A. These treatment management led to reduction in seizures frequency or even to seizures remission in some cases.

Conclusions The recognition and diagnostic of underlying etiologies of intractable seizures improve the treatment management in many cases. The excessive NMDA transmission might be the part of pathogenesis of seizures in children with inborn error of metabolism.

\section{GP233 LIFESAVING MECHANICAL THROMBECTOMY IN PAEDIATRIC STROKE}

${ }^{1}$ Emily Farnan*, ${ }^{1} \mathrm{MP}$ O'Riordan Stephen, ${ }^{2}$ Noel Fanning, ${ }^{2}$ Gerald Wyse, ${ }^{3}$ Clodagh Ryan, ${ }^{4}$ Niamh Mc Sweeney. ${ }^{1}$ Department of Paediatrics and Child Health, Cork University Hospital, Cork, Ireland; ${ }^{2}$ Interventional Neuroradiology, Cork University Hospital, Cork, Ireland; ${ }^{3}$ Department of Paediatric Haematology, Mercy University Hospital, Cork, Ireland; ${ }^{4}$ Paediatric Neurology, Cork University Hospital, Cork, Ireland

10.1136/archdischild-2019-epa.292

Introduction Childhood arterial ischaemic stroke (AIS) is uncommon with a reported incidence between 1.2 and 7.91 per 100,000 per year. ${ }^{1,2,3}$ Previously it was thought that children with AIS had a good outcome due to brain plasticity; however, mortality has been reported in up to $28 \%$, and morbidity in up to $70 \%$ of survivors. ${ }^{4,5}$ There are no randomised trials of mechanical thrombectomy in children. The 2017 published RCPCH stroke guidelines draw on the excellent outcomes for mechanical thrombectomy in adult trials and recommend referral for intra-arterial clot extraction in patients with NIHSS score of 6 or more and up to 12 hours post onset if there is salvageable brain tissue on imaging. ${ }^{6}$ There are only 29 paediatric cases published in the literature that have undergone mechanical thrombectomy, 12 of which were for posterior circulation AIS. 